\title{
Cardiac muscle organization revealed in 3-D by imaging whole-mount mouse hearts using two- photon fluorescence and confocal microscopy
}

Mayandi Sivaguru, Glenn Fried¹, Barghav S. Sivaguru², Vignesh A. Sivaguru², Xiaochen Lu³, Kyung Hwa Choi ${ }^{4}$, M Taher A Saif ${ }^{4}$, Brian Lin ${ }^{5}$, and Sakthivel Sadayappan ${ }^{5}$

${ }^{1}$ Microscopy and Imaging Core Facility, Carl R. Woese Institute for Genomic Biology, ${ }^{2}$ College of Liberal Arts and Sciences, ${ }^{3}$ Department of Cell and Developmental Biology and Carl R. Woese Institute for Genomic Biology, ${ }^{4}$ Department of Mechanical Science and Engineering, University of Illinois at Urbana Champaign, Urbana, and ${ }^{5}$ Cell and Molecular Physiology, Stritch School of Medicine, Loyola University, Maywood, IL

BioTechniques 59:295-308 (November 2015) doi 10.2144/000114356

Keywords: whole-mount; heart-3-D; MYBPC3; PAS labeling; heart failure; two-photon; confocal microscopy

Supplementary material for this article is available at www.BioTechniques.com/article/114356.

The ability to image the entire adult mouse heart at high resolution in 3-D would provide enormous advantages in the study of heart disease. However, a technique for imaging nuclear/cellular detail as well as the overall structure of the entire heart in 3-D with minimal effort is lacking. To solve this problem, we modified the benzyl alcohol:benzyl benzoate (BABB) clearing technique by labeling mouse hearts with periodic acid Schiff (PAS) stain. We then imaged the hearts with a combination of two-photon fluorescence microscopy and automated tile-scan imaging/stitching. Utilizing the differential spectral properties of PAS, we could identify muscle and nuclear compartments in the heart. We were also able to visualize the differences between a 3-month-old normal mouse heart and a mouse heart that had undergone heart failure due to the expression of cardiac myosin binding protein-C (cMyBP-C) gene mutation (t/t). Using 2-D and 3-D morphometric analysis, we found that the $t / t$ heart had anomalous ventricular shape, volume, and wall thickness, as well as a disrupted sarcomere pattern. We further validated our approach using decellularized hearts that had been cultured with 3T3 fibroblasts, which were tracked using a nuclear label. We were able to detect the 3T3 cells inside the decellularized intact heart tissue, achieving nuclear/cellular resolution in 3-D. The combination of labeling, clearing, and two-photon microscopy together with tiling eliminates laborious and time-consuming physical sectioning, alignment, and 3-D reconstruction.

Past advances in imaging technologies have led to multiple methodologies for imaging intact hearts (1-6). Echocardiography (7), ultrasound-biomicroscopy $(1,2)$, magnetic resonance imaging (MRI) (1-4), micro computed tomography (micro-CT) (1-4), and confocal microscopy $(5,6)$ are common techniques used for whole heart or embryo imaging. While most of these methods do not produce nuclear/cellular resolution, 3-D reconstruction using confocal microscopy is economical, reasonably fast, and provides cellular or nuclear resolution as well as revealing cardiac muscle organization. However, recent advances in micro-CT $(3,4)$ allow for submicron resolutions.
Pioneering efforts have used episcopic imaging of autofluorescence in the sample block face of intact small embryos and hearts to produce highresolution 3-D reconstructions (8-10). Ten years ago, a similar approach was developed by Miller et al. using two-photon confocal microscopy of whole mouse hearts (5). By combining

\section{METHOD SUMMARY}

Here we present a procedure to generate high-resolution 3-D quantifiable images of intact mouse hearts. Following perfusion with NOTOXhisto, hearts were dehydrated, cleared using benzyl alcohol:benzyl benzoate (BABB), rehydrated, stained with periodic acid Schiff (PAS), and then dehydrated and cleared again. Labeled hearts were then mounted in BABB in a custom sample holder and imaged using two-photon fluorescence or single-photon confocal microscopy. Stitching lines between frames were removed by custom MATLAB code, while 3-D composite, rendering, and volume measurements were performed with Bitplane Imaris software. 
benzyl alcohol:benzyl benzoate (BABB) clearing with two-photon and confocal microscopy (without automated tiling), a depth of up to $1.5 \mathrm{~mm}$ was reached in chicken embryos and mouse embryos. Smith et al. (6), using the same BABB technique, revealed angular variation in heart muscle orientation using a membrane dye in intact hearts. Miller and colleagues enhanced the nuclear contrast by labeling with several nuclear dyes, but the muscle was not labeled to increase the contrast. Smith et al. achieved muscle contrast using a membrane dye, but only single-photon microscopy was employed, allowing just half the depth of penetration. In both cases, the heart was rotated physically as needed to cover the depth, and lower power objectives were used to cover the entire heart in a single field of view without tiling. After taking a Z-stack halfway through one side and by rotating the sample physically, Smith and colleagues were able to achieve an imaging depth of $3.5 \mathrm{~mm}$. The aforementioned techniques as well as the techniques presented in this paper

Table 1. Estimated time required for heart clearing, labeling, 3-D rendering, visualization, and analysis.

\begin{tabular}{|c|c|}
\hline Procedure & Approximate time required \\
\hline Fixation & $1-2 \mathrm{~h}$ \\
\hline Dehydration (ethanol series, $25 \%, 50 \%, 75 \%$, and $100 \%$ ) & $2-3 \mathrm{~h}$ \\
\hline Ethanol:BABB ratios $(2: 1,1: 1,1: 2)$ h each & $6 \mathrm{~h}$ (12 h if parked in one step overnight) \\
\hline Clearing with $\mathrm{BABB} 3 \times$ & 45 min-2 h (depending on the sample size) \\
\hline Rehydration (ethanol series, 100 to $25 \%$ ) & $2-3 h$ \\
\hline Staining with PAS and nuclear dye (if needed) & $30 \mathrm{~min}$ \\
\hline Dehydration (ethanol Series, $25 \%-100 \%$ ) & $2-3 h$ \\
\hline Ethanol:BABB ratios $(2: 1,1: 1,1: 2) 2 \mathrm{~h}$ each & $6 \mathrm{~h}$ (12 h if parked in one step overnight) \\
\hline Clearing with $\mathrm{BABB}^{1}$ & $45 \mathrm{~min}-2 \mathrm{~h}$ (depending on the sample size)* \\
\hline $\begin{array}{l}\text { Two-photon imaging of entire heart with four line scan } \\
\text { average*** }\end{array}$ & $\sim 3.8-4.5 h^{* * *}$ \\
\hline $\begin{array}{l}\text { Imaris volume tracing every } 40-50 \mu \mathrm{m} \text { through the entire } \\
\text { depth (making } 80-100 \text { traces for each ventricle/heart) }\end{array}$ & 45-60 min/ventricle \\
\hline $\begin{array}{l}\text { 2-D morphometric measurements on median plane; at least } \\
\text { four measurements per parameter per heart }\end{array}$ & $45-60 \mathrm{~min} /$ heart \\
\hline $\begin{array}{l}\text { 3-D rendering/ visualization (high resolution shots and movie } \\
\text { making) and 2-D and 3-D morphometric analysis }\end{array}$ & $1-2 h^{2}$ \\
\hline \multicolumn{2}{|c|}{$\begin{array}{l}\text { *If air bubbles form during this step, keep sample in vacuum for a couple of hours to remove bubbles. } \\
{ }^{* *} \text { Other parameters such as dimensions and image size are provided in Table } 2 \text {. } \\
\text { *** The calculation of image acquisition time depends on the available RAM in the computer running } \\
\text { the Zen software (i.e., depending on the image and data size, the actual time could be longer than the } \\
\text { estimated time.) } \\
{ }^{1} \text { Fresh BABB solution is preferred in the last clearing step. } \\
{ }^{2} \text { Actual time depends on computer hardware resources, graphics processing unit RAM, and number of } \\
\text { processors in the system. }\end{array}$} \\
\hline
\end{tabular}

carry trade-offs between resolution, efficiency, optimal sampling frequency (Nyquist rate), speed, and cost, based on which dyes, hardware, software, and techniques are used. However, the optics employed are similar in both the Miller et al. and Smith et al. works $(5,6)$. We attempted to improve upon

\section{Sometimes it takes more than one slice to make a great sandwich.}

\section{The SageELF ${ }^{\mathrm{TM}}$ from Sage Science.}

\section{Collect multiple slices from a single sample.}

\section{Automated Gel Purification for: \\ - Next Gen Sequencing \\ - Protein Mass Spec}

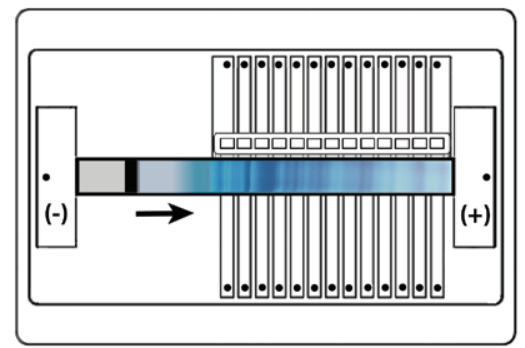

Separation
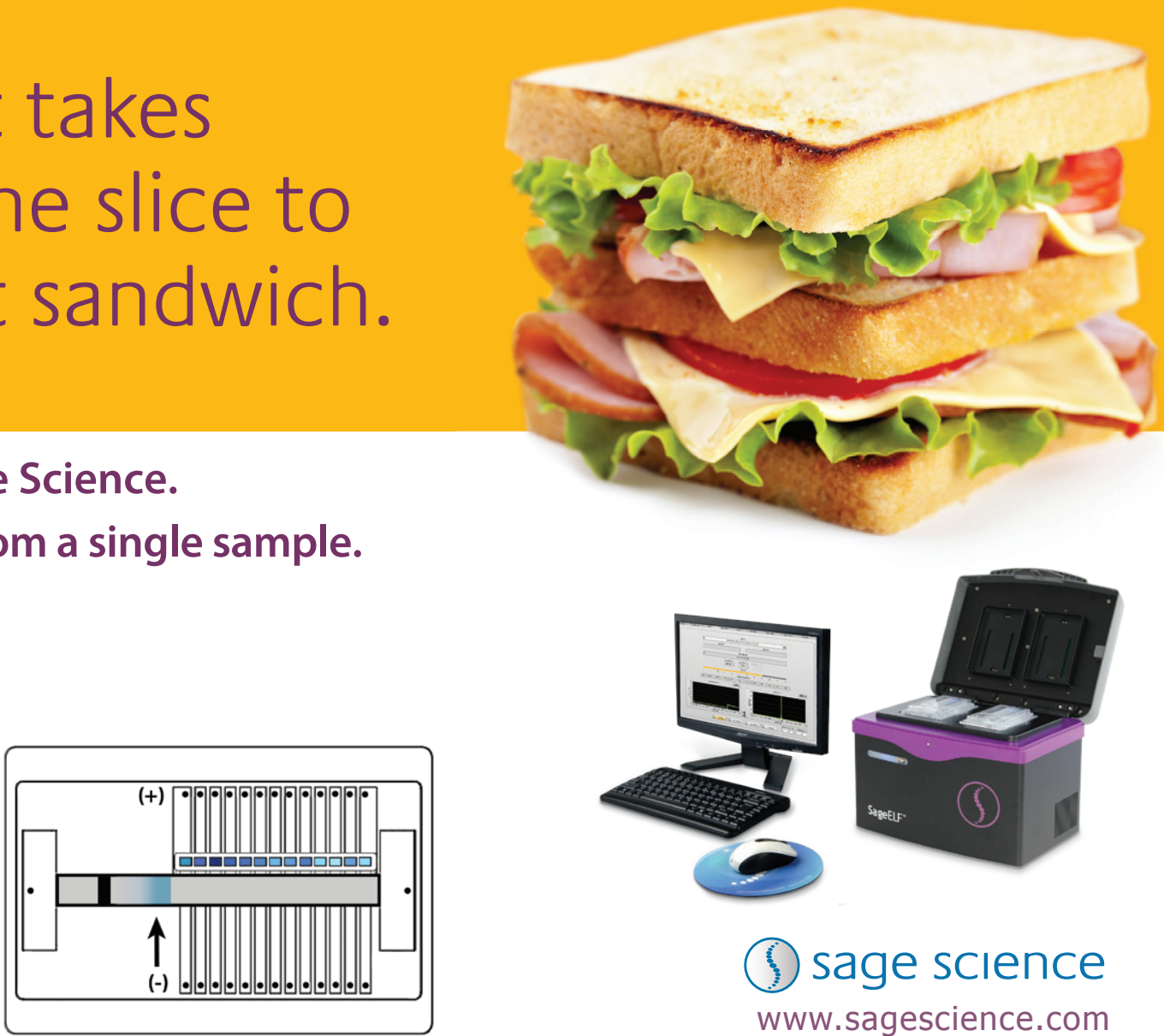

sage science www.sagescience.com

Fractionation 
all aspects of the former approaches, such as enhancing muscle and nuclear contrast, dual imaging with both single- and two-photon techniques, and obviating the need for physical sectioning and rotation of the sample.

To demonstrate the efficacy of this modified two-photon fluorescence microscopy technique in the present study, we first used a homozygous mouse model of dilated cardiomyopathy (DCM) due to the C-terminal truncation mutation in the cardiac myosin binding protein- $C$ (cMyBP-C) gene (t/t) (11) and compared this to a normal mouse heart. CMyBP-C is important for maintaining myofibrillar integrity and basal contractile function $(12,13)$. Expression of $t / t$ was functionally compromised at the whole-heart level, showing impaired hemodynamics and severe DCM at the age of 3 months (11). The homozygous $t / t$ mice were viable and fertile, but soon after birth displayed progressive heart failure with DCM (11). The $t / t$ hearts showed markedly enlarged ventricular chambers, with significant myocyte disarray and fibrosis compared with normal control hearts. Importantly, there are several studies that have extensively used the $t / t$ mouse model to study the severe DCM phenotype (12,14-16), suggesting the $t / t$ mouse heart is an ideal model to test the present technique.

In the second example, to further evaluate the usefulness of this technique we used a set of hearts that were going to be used as scaffolds or substrates for cultured cells in regenerative medicine studies. Tremendous efforts $(17,18)$ have been made to understand cellular behavior on 2-D and 3-D substrates coated with common extracellular matrix (ECM) molecules such as fibronectin and collagen in vitro. However, unless new biomaterials that closely mimic natural ECM components are made and readily available, obtaining reliable results that approximate actual cell behaviors in vivo continues to be a challenge. Therefore, tissue engineers have turned to acellular organ/tissue-derived matrices, using them as 3-D substrates into which labeled cells are replanted and cellular behaviors, including reshaping of ECM structure, can be observed. Although this idea is not new in the fields of tissue engineering and regenerative medicine, imaging the whole organ/tissue without sectioning has been always one of the biggest challenges. Because of this, we tried to apply our new imaging method to obtain a complete 3-D structure of murine hearts with and without cultured cells inside the heart scaffold, as this has potential to be utilized in cell behavior studies such as cancer research.

Here we present a straightforward and minimal-effort visualization and 3-D quantification procedure customdeveloped for heart imaging at nuclear and sarcomere resolution. The protocol uses Murray's clearing solution (also known as BABB clearing solution) (19-21) in tandem with periodic acid Schiff (PAS) stain, which binds glycogen and lightly stains nuclei (21-23). We used two-photon imaging, which allows greater depth of penetration in a 3-D sample (24) as the out of focus excitation is totally eliminated because of the two-photon phenomena (24). Since only the muscle is excited at a given wavelength, penetration over $\sim 3.5 \mathrm{~mm}$ along the z-axis was possible, with this limitation only imposed by the working distance of the objective. Using a single-photon laser in a confocal system at specific wavelengths allowed discrimination of glycogen and nuclei localization in the same samples. The results are presented in the context of using this technique for intact tissues and organs; not just for visualization, but also for a multitude of high-resolution 2-D and 3-D measurements, analyses, and quantification.

\section{Materials and methods}

Experimental samples, cannulation, perfusion, and surgical procedures

The $\mathrm{t} / \mathrm{t}$ mouse model was originally generated in the Seidman laboratory at Harvard Medical School $(11,15)$, and t/t mouse lines in the $\mathrm{FvB} / \mathrm{N}$ background were available from and maintained at

Table 2. Image acquisition parameters, dimensions, duration, resolution limits, and image size.

\begin{tabular}{|c|c|c|c|}
\hline Parameter & NTG & $t / t$ & Scaffolds \\
\hline $\begin{array}{l}\text { Voxel dimension two-photon 3-D } \\
\text { images }(10 \times)\end{array}$ & $\begin{array}{l}\text { Voxel Size: } X: 2.786, Y: 2.768, Z: 20 \\
\mu m ; \text { Image: } 6 \times 4 \times 148 \text { ( } 24 \text { tiles and } \\
3552 \text { images) } \\
\text { Image dimension: } X: 8499, Y: 5665 \\
Z: 2960 \mu \mathrm{m}(0.87 \mathrm{~GB} \text { size) }\end{array}$ & $\begin{array}{l}X: 2.786, Y: 2.768, Z: 20 \mu \mathrm{m} / \text { pixel; } \\
\text { Image: } 6 \times 5 \times 176(30 \text { tiles and } 5280 \\
\text { images) } \\
\text { Image dimension } X: 8499, Y: 7082 \\
Z: 3500 \mu \mathrm{m}(1.3 \mathrm{~GB} \text { size) }\end{array}$ & $\begin{array}{l}\mathrm{X}: 2.0, \mathrm{Y}: 2.0, \mathrm{Z}: 20 \mu \mathrm{m} / \text { pixel; } 6 \times 4(24 \\
\text { tiles, varying } \mathrm{Z} \text { depths and file size*) } \\
\text { Image dimension } \mathrm{X}: 6374, \mathrm{Y}: 4248, \\
\mathrm{Z}: \text { variable* }^{*} \text { (size variable and }<1 \mathrm{~GB} \text { ) }\end{array}$ \\
\hline $\begin{array}{l}\text { Pixel dimension two-photon 2-D images } \\
\text { High-resolution images }(25 x)\end{array}$ & \multicolumn{3}{|c|}{$\begin{array}{l}0.554 \mu \mathrm{m} \text { for swelling and } \sim 0.111 \mu \mathrm{m} \text { for sarcomere images using the oil immersion objective }(25 \times / 0.8 \mathrm{LCl} \text { LD Plan Neofluar } \\
\text { Korr DIC) }\end{array}$} \\
\hline $\begin{array}{l}\text { Voxel dimension single-photon } \\
\text { multichannel images }\end{array}$ & \multicolumn{3}{|c|}{$\begin{array}{l}0.781 \text { to } 1.25 \mu \mathrm{m} \text { depending on single or tiled images }(10 \times) \\
0.313 \text { to } 0.625 \mu \mathrm{m} \text { depending on single or tiled images }(20 \times)\end{array}$} \\
\hline Dichroic, emission filter, $780 \mathrm{~nm} * *$ & \multicolumn{3}{|c|}{ 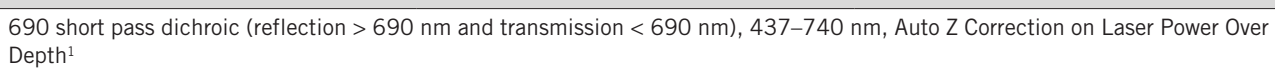 } \\
\hline Dichroic, emission filter, $488 \mathrm{~nm}$ & \multicolumn{3}{|c|}{ MBS 405/488/555/633, EF 497 Long Pass VSD*** } \\
\hline Dichroic, emission filter, $555 \mathrm{~nm}$ & \multicolumn{3}{|c|}{ MBS 405/488/555/633, EF 561 Long Pass VSD*** } \\
\hline $\begin{array}{l}\text { Pixel dwell time / scan time per frame / } \\
\text { scan time per image }\end{array}$ & $1.58 \mu \mathrm{s} / 3.87 \mathrm{~s} / 3.81 \mathrm{~h}^{2}$ & $1.58 \mu \mathrm{s} / 3.87 \mathrm{~s} / 5.67 \mathrm{~h}^{2}$ & $1.58 \mu \mathrm{s} / 3.87 \mathrm{~s} /$ variable $^{2}$ \\
\hline $\begin{array}{l}\text { Theoretical resolution and working } \\
\text { distances (WD) of objectives used }\end{array}$ & \multicolumn{3}{|c|}{ 10x: $1.11 \mu \mathrm{m}(5.5 \mathrm{~mm}$ WD), 20×: $0.42 \mu \mathrm{m}(0.55 \mathrm{~mm}$ WD), $25 \times$ LD Oil: $0.42 \mu \mathrm{m}(0.57 \mathrm{~mm}$ WD) } \\
\hline \multicolumn{4}{|c|}{$\begin{array}{l}\text { *The control heart, decellularized heart and decellularized heart seeded with cells had different Z depths; see Supplementary Material for raw data "Information" } \\
\text { screen shots for independent dimensions. } \\
* * \text { The spectral range reflects the entire emission spectra collected for PAS, see Figure } 4 \text { for spectral profile and emission peaks under single and two-photon } \\
\text { excitation. } \\
\text { *** Long pass variable secondary dichroic (VSD) used in LSM } 700 \text {. } \\
{ }^{1} \text { The auto Z correction is a modality available in Zen software to linearly extrapolate the laser power over the given range of Z (see Supplementary Material } \\
\text { information screen shot for actual laser power ranges). } \\
{ }^{2} \text { Estimated time; could be longer depending on system resources. }\end{array}$} \\
\hline
\end{tabular}


Figure 1. High-resolution whole-mount imaging and visualization of the entire mouse heart. (A) NOTOXhisto-fixed fresh heart (in PBS), dehydrated and cleared in benzyl alcohol:benzyl benzoate (BABB) (Cleared) showing the level of transparency, and the merge of both images showing the level of shrinkage after processing (* indicates edge of the unprocessed fresh heart). (B) Two-photon fluorescence microscopy (TPFM) of BABB-cleared and periodic acid Schiff (PAS)-stained heart showing a single, tiled optical section (from a total of 3600 images with the image dimension in $X Y Z$ of $8.5 \times 5.7 \times 3.0 \mathrm{~mm}$ respectively) with lines in the tile regions (left and zoomed inset at the bottom with arrows pointing the lines), which were removed using custom developed MATLAB code (right and zoomed in at the bottom). (C) Details of the well-preserved myocardial internal structures, as all veins and blood vessels (arrows) are revealed. (D) Confocal single-photon imaging of the same BABB-cleared and PAS-labeled hearts at the indicated wavelengths showing the separation of muscle (green) and nuclear (red) locations (Panel 1), gallery of images (excitation wavelength $488 \mathrm{~nm}$ ) in black and white showing the orientation (see also the zoomed-in images on the right) of muscle through the $z$-axis (Panel 2), and an $\mathrm{XZ}$ projection of the single-photon 488 $\mathrm{nm}$ image showing the limitation of depth penetration (Panel 3), compare the with two-photon excitation shown in (E). (E) Orthogonal sections ( $X Y, X Z$, and $Y Z$ ) of the whole heart at the median plane showing the left and right ventricle dimensions. Scale bars in (A), (B), and (D) are $1 \mathrm{~mm}$; bars in (C) are 100 and 200 microns for the left two images and the right one, respectively; bars in D represent 100 microns.
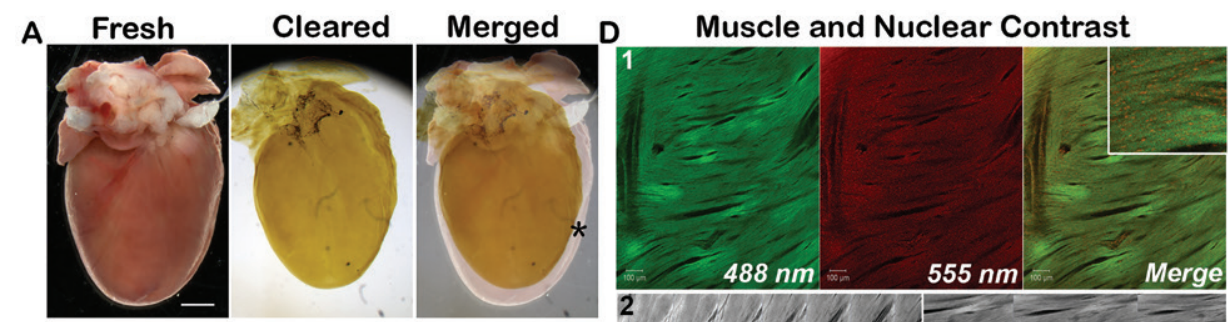

B Tile Scan Lines

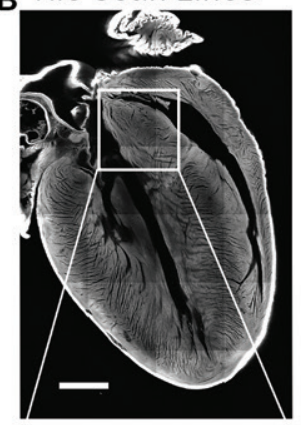

Lines removed
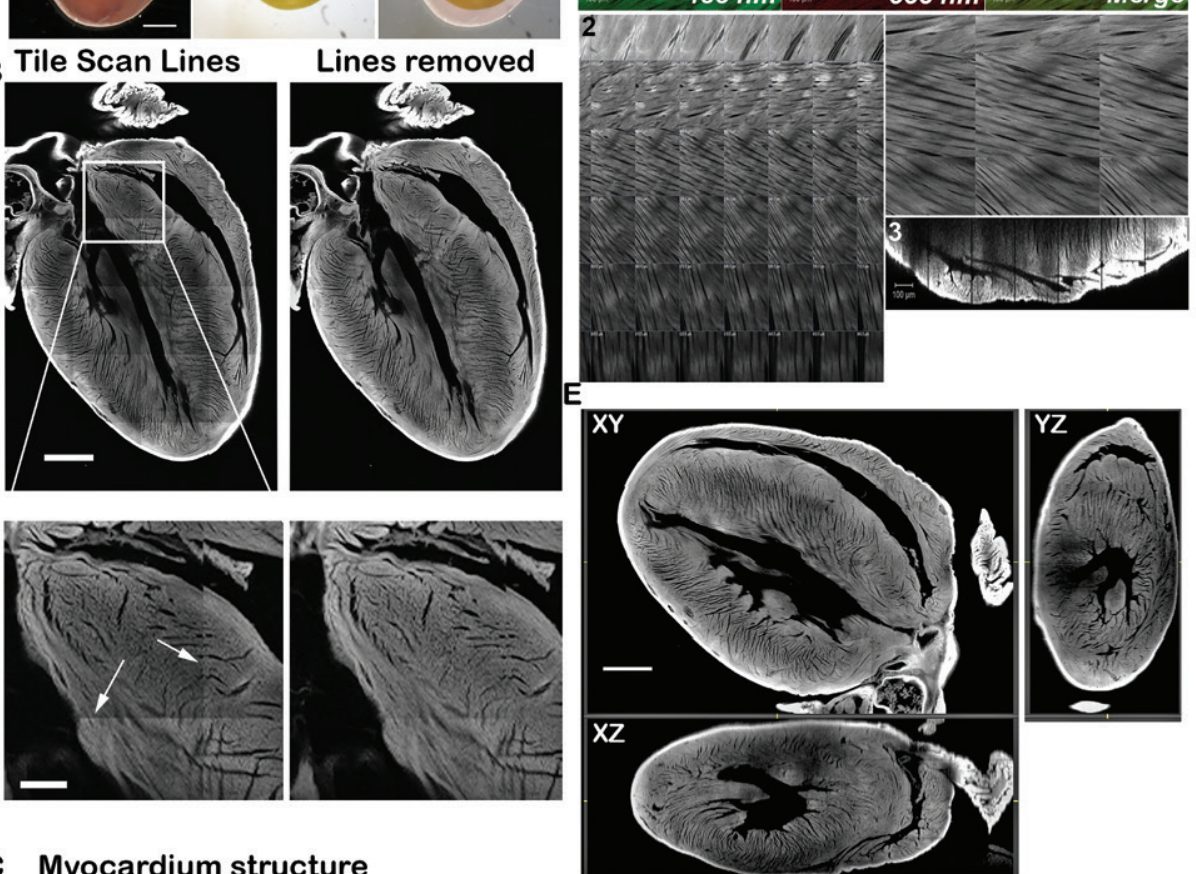

C Myocardium structure

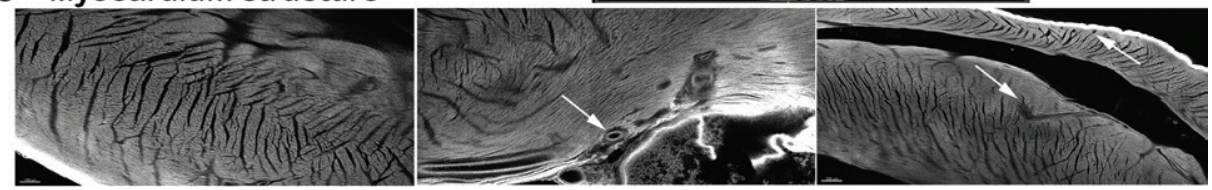

Loyola University of Chicago $(11,16)$. Three-month-old normal and t/t adult mice hearts were heparinized (in $0.25 \mathrm{~mL}$ 1000 USP/mL heparin sodium) (Sagent Pharmaceuticals, Schaumburg, IL) to prevent blot clots from forming during the removal process. Mice were then anesthetized with 5\% isoflurane (Abbott Animal Health, Abbott Park, IL) in medical grade oxygen. Animals were determined to be unconscious by the lack of a reflex response from rear toe pinches.

To ensure optimal cardiac tissue integrity, the following steps must be conducted in rapid and deliberate fashion: The chest must be cut from below the diaphragm, and the rib cage must be cut on each side so that it can be pulled back to expose the heart. The heart is rapidly excised, with care taken to ensure the aorta remains attached. The heart is then cannulated via the aorta with a blunt syringe needle and affixed with a bulldog clamp. The heart is then perfused with approximately $5 \mathrm{~mL}$ ice-cold cardioplegic solution (50 mM KCl, 5\% dextrose, $1 \times$ PBS) to relax the cardiac muscle completely. The blood in the coronary arteries will be visibly flushed out of the heart, and the heart will then have a consistent pink coloration. Once this occurs, the heart is perfused with $5 \mathrm{~mL}$ ice-cold NOTOXhisto fixative (Science Device Laboratory, Des Plaines, IL). The present experiments were approved by the Institutional Animal Care and Use Committees at Loyola University in Chicago and the University of Illinois at Urbana-Champaign, following the National Institutes of Health Guide for the Use and Care of Laboratory Animals.

\section{Decellularization procedure for heart scaffolds}

For the heart-as-scaffold experiments, three whole hearts were obtained from neonatal rats: one as a control, one as a decellularized scaffold, and one as a decellularized scaffold seeded with 3T3 fibroblasts. The control heart was kept in PBS until it underwent the clearing and staining processes. All of the cells in the remaining two hearts were extracted following the decellularization protocol (18). Briefly, the hearts were kept in distilled water at $4^{\circ} \mathrm{C}$ for 24 $\mathrm{h}$. The hearts were then immersed in the solution containing $0.5 \%$ Triton $\mathrm{X}-100$ and $0.05 \%$ ammonium hydroxide for 72 h. Subsequently, the decellularized hearts were immersed in PBS at $4^{\circ} \mathrm{C}$. One of the decellularized hearts was soaked in warm 3T3 fibroblast medium (high-glucose DMEM with $4 \mathrm{mM}$ L-glutamine, 10\% fetal bovine serum, $1 \%$ penicillin-streptomycin) at $37^{\circ} \mathrm{C}$ for $20 \mathrm{~min}$ before cell seeding. After $20 \mathrm{~min}$, this heart was transferred to a 14-mm-well glass bottom Petri dish. Subsequently, $500 \mu \mathrm{L}$ cell suspension solution containing $1.4 \times 10^{6}$ cells was 

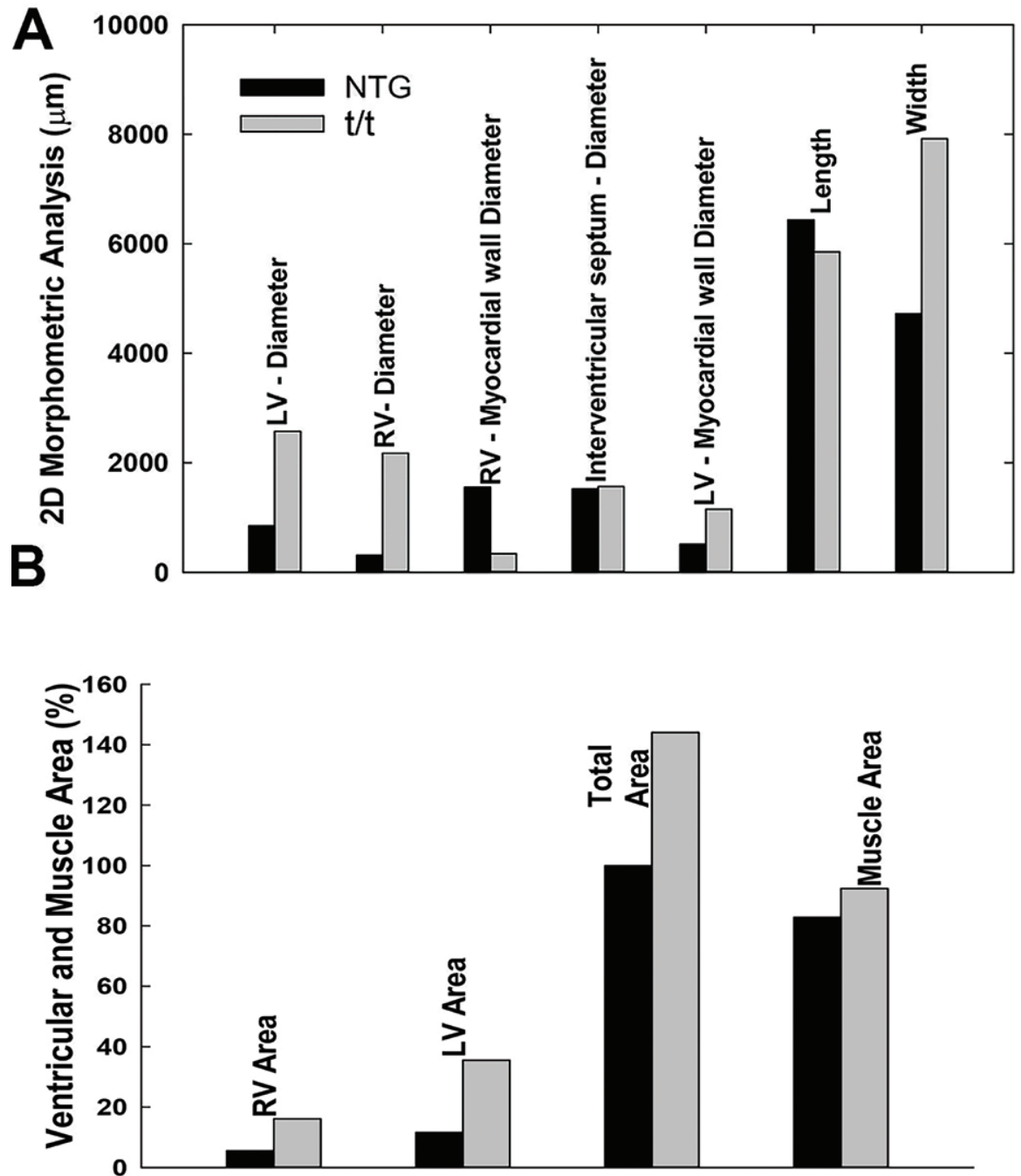

Figure 3. 2-D Analysis of normal and $t / t$ heart dimensions. Morphometric analysis ( $A$ ) of whole heart dimensions and the area of the ventricles (B) at the median plane for the normal and t/t hearts using a 2-D interactive measurement module.

added to the well and incubated at $37^{\circ} \mathrm{C}$ and $5 \% \mathrm{CO}_{2}$ for $30 \mathrm{~min}$. After that, $2 \mathrm{~mL}$ of the warm medium was added, and the sample was kept in the incubator for 1 day. After $24 \mathrm{~h}$, the medium was replaced with fresh medium to remove any non-adherent cells. Finally, the hearts were harvested and transferred to NOTOXhisto fixative immediately and fixed for $2 \mathrm{~h}$, with the initial 15 min under house vacuum.

\section{Clearing and labeling procedures}

We dehydrated each heart in a graded ethanol series $(25 \%, 50 \%, 75 \%$, and $100 \%$ in PBS under vacuum). Dehydrated hearts were taken through increasing BABB-to-ethanol ratios $(1: 2,1: 1,2: 1)$, and placed 3 times in absolute BABB until the tissue sank and the heart was visibly clear. The approximate duration for each step in the procedure is listed in Table 1. The hearts were then rehydrated in a decreasing ethanol series and labeled with a Periodic Acid-Schiff (PAS) kit (SKU 395B, Sigma-Aldrich Corp., St. Louis, MO), according to the manufacturer's protocol, until they turned translucent purple. They were then washed in water (5-10 min), and stained with 1000 $\mathrm{ng} / \mathrm{mL}$, Hoechst 33342 (control, decellularized, and decellularized seeded with cells in scaffold experiments) and a nuclear counterstain (Life Technologies, Eugene, OR) under vacuum for $1 \mathrm{~h}$. We then washed each heart and repeated the dehydration and $\mathrm{BABB}$ series as above, ending with $3 \times 100 \%$ BABB washes. Total time was approximately 25-30 $\mathrm{h}$ depending on the sample size, tissue type, and clearing speed. As shown in Figure 1, there was an isotropic shrinkage of $~ 6.1 \%$ (width) and $~ 8.8 \%$ (length) of the BABBcleared heart compared with when it was in aqueous fixative. These images were obtained in a Zeiss Axiozoom V16 microscope with a Plan Neofluar 1× 0.25 NA objective (Carl Zeiss Inc., Jena, Germany). Cleared and labeled hearts were mounted in a custom-made cover glass-bottom dish (\#1.5 or 0.17 $\mathrm{mm}$ ) to which a glass tube was glued (see Supplementary Material). BABB dissolves most kinds of plastic; care should be taken to prevent leakage or spilling to protect optics and equipment. We adjusted the diameter of the glass tube and the height of the BABB solution, and a special type of glue was used to seal the cover glass and glass tube (Loctite, Prism, 46040 from Electron Microscopy Sciences, Hatfield, PA). The top chamber was closed with another cover glass during imaging to prevent any evaporation and draftinduced tissue movement.

\section{Confocal and two-photon} fluorescence microscopy

We imaged heart samples on a Zeiss LSM 710 NLO system with a two-photon laser (Mai Tai eHP with DeepSee; Newport Corporation, Irvine, CA), 488 and $561 \mathrm{~nm}$ single photon lasers with a regular photomultiplier tube or a 32 channel quasar spectral detector to characterize the emission peaks for PAS- and Hoechst-stained samples. The $780 \mathrm{~nm}$ excitation was used to exploit the peak power of the two-photon laser so that it would give a better penetration depth, and the PAS dye was excited with this wavelength adequately. In addition, in the scaffolds, we used Hoechst nuclear dye to achieve better penetration depth, and although $780 \mathrm{~nm}$ is not the optimal exitation wavelength for the dye, we could detect the nuclei in the control scaffold with this wavelength. In addition, we used a Zeiss LSM 700 system with 488 and 555 $\mathrm{nm}$ lasers for single-photon microscopy tiling. The images were obtained using either a 10x EC Plan Neofluar 0.3 NA (working distance $5.5 \mathrm{~mm}$ ) or a 20x Plan Apochromat 0.8 NA (working distance $0.55 \mathrm{~mm}$ ) or a $25 \times$ LD LCl Plan Neofluar 0.8 NA Korr DIC (working distance 0.57 $\mathrm{mm}$ ) used with oil immersion (all objec- 

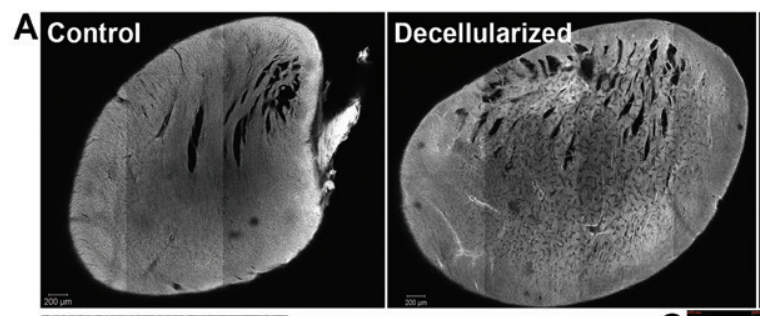

Decellularized cultured
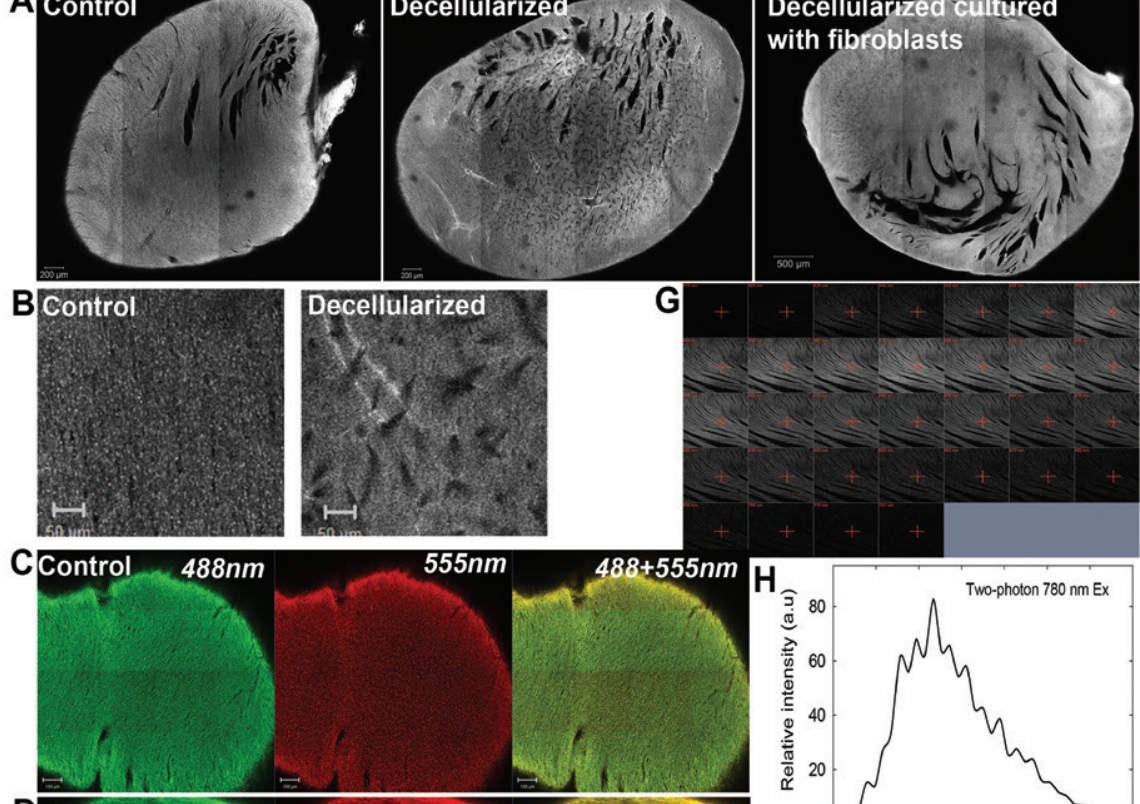

Decellularized

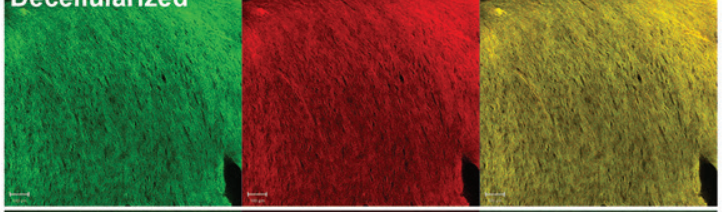

EDecellularized cultured with fibroblasts-Surface plane
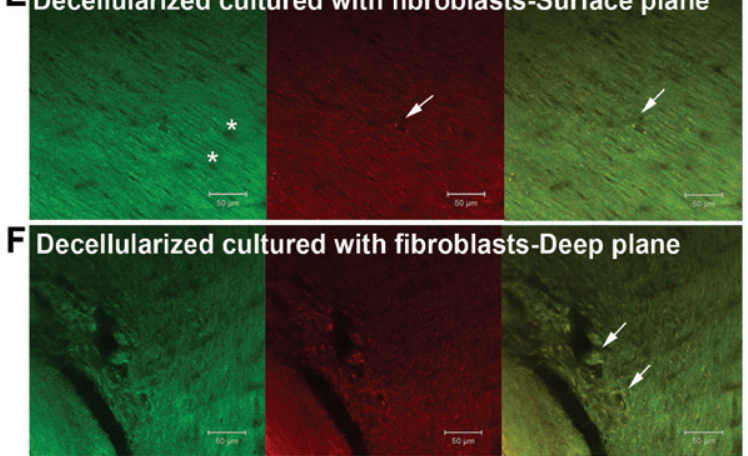

Figure 4. Decellularized hearts as scaffolds and spectral properties of PAS. (A) Two-photon $(780 \mathrm{~nm})$ excitation of heart scaffolds labeled with both PAS and Hoechst nuclear dye sequentially, and cleared with BABB. (B) High magnification images of the control and decellularized hearts show the nuclear contrast dye in the control and the absence of nuclei in the decellularized hearts. (C-F) Single-photon images with the indicated excitation wavelengths of the same scaffolds labeled with both PAS and Hoechst nuclear dye showing spotty nuclei in the control (C), no nuclear presence in decellularized heart (D), but sporadic presence of nuclei on the surface (E) within deeper ( 100 micron) focal planes (F) of a decellularized heart (note the holes below the asterisks from where cells were removed during the decellularization process) cultured with 3T3 fibroblast cells. (G) Two-photon $(780 \mathrm{~nm}$ ) spectral cross section showing the PAS dye emission spectral profile from 419-721 nm (each box represents a 10 micron step). (H) The entire emission spectral profiles of the entire emission spectrum using both single- and two-photon excitation at the indicated wavelengths. Note the broad peak with two-photon and peak shifts from 550 to $630 \mathrm{~nm}$ under 488 and $561 \mathrm{~nm}$ excitation, respectively. Scale bars in (A): first 2 images-200 microns, third image-500 microns; (B), (E), and (F): 50 microns; (C) and (D): 100 microns.

tives are from Carl Zeiss Inc.). Details of each image acquisition parameter (including laser power and gain settings) are provided in the Supplementary Material as well as in Table 2. Briefly, we used an automated $\mathrm{XY}$-stage and $z$-axis focus to image $24-30,10 \times(0.6$ zoom) fields at a 10 or 20 micron step size along the $z$-axis and a 2.8 micron pixel size in XY (see Supplementary Material and Table 2). We used the Zeiss "Auto Z-Correction" option in the Z-stack module, where the laser power could be linearly amplified from the bottom of the image to the top of the image (i.e., to compensate for the loss of excitation light due to absorption and scattering in such a large sample). For whole-heart imaging with two-photon microscopy, the images are undersampled to obtain a compromise among speed of acquisition, size of the data, and prevention of photobleaching; however Nyquist sampling was possible and was mostly met in single-photon images, where tiles are not necessary. Fulfilling the Nyquist sampling criteria could lead to data sets that are 100 times larger, so the undersampling was done for practical purposes, but it does not mean that we cannot meet the Nyquist sampling rate. When computers become powerful enough to handle such data sets, we should be able to collect data meeting the Nyquist rate, which would provide phenomenal resolutions using this technique. The images are stitched with $0 \%$ overlap in the native Zeiss 2 Black Edition software.

\section{2-D and 3-D image}

\section{analysis and rendering}

2-D image calculations were performed with the Zeiss Axiovision software, where the morphometric measurements such as length, width, height, area of ventricles, and muscle and myocardial wall dimensions were made for four different locations for each parameter, and the average values were presented. Whole-heart and ventricular volumes for normal and $t / t$ hearts (see Supplementary Material) were traced (every 40-50 micron step through the z-axis) using the Surpass module (Contour Tracing Algorithm) in the Imaris Suite (25) software (version 8.0; Bitplane, Inc., Zurich, Switzerland). The whole-heart volume was created using the Isosurface module in the same Imaris program after applying background subtraction, smoothing and a threshold for the muscle based on the intensity of muscle volume. Highresolution snapshots and movies were made in the same program using key frame animation, and the resolution of the movies was reduced to keep the file sizes low. Final images and their intensities were adjusted at $\mathrm{min} / \mathrm{max}$ display settings to best represent the dynamic range of the gray values of a given image. Images were assembled 
in Adobe Photoshop (Adobe Systems Inc., San Diego, CA). We developed a custom MATLAB (version 2012b; The MathWorks, Natick, MA) routine to remove tile scan seams (Figure 1) in hearts used for volume rendering and 3-D measurements (The full functional code is included in the Supplementary Material).

\section{Results and discussion}

We achieved remarkable clarity, resolution, and quantifiable contrast for the entire mouse heart using this technique, without having to rotate sides (Figures 1-4). Figure 1A shows the raw control non-transgenic mouse heart after excision and the impact of clearing and labeling. BABB clearing caused no observable shrinkage. Although BABB has been reported to produce higher levels of shrinkage (5), it is possible that the age and exact stage of the heart muscle might affect shrinkage as well. The tiled heart images and the efficacy of seam removal in MATLAB (see a full resolution Z-stack in Supplementary Movie S1) is shown in Figure 1B (see the full MATLAB code in the Supplementary Material). Figure $1 \mathrm{C}$ shows details of myocardial muscle architecture typically observed in thin sections of hematoxylin and eosin (H\&E) stained samples (7), with locations of veins and intermuscular junctions representing fine muscle architecture. With single-photon imaging at two different wavelengths, we could distinguish glycogen/muscle (488 $\mathrm{nm}$ ) and individual nuclei (561 $\mathrm{nm}$ ) using the same PAS dye, where the nuclear contrast is greater due to clear separation of glycogen and nuclear emission peaks (Figure 1D and Figure $4 \mathrm{H}$ ). Compared with the technique of Smith et al. (6), where the muscle angles in intact hearts were measured using a membrane dye, we believe that labeling with PAS produces a much more robust muscle contrast, which allows the depthdependent change in the angle to be more precisely measured (Figure 1D, panel 2). Also, because of the nuclear contrast in single-photon imaging, this technique allows easy quantification of nuclear density anywhere in the heart (Figure 1D, panel 1). However, as predicted by theory (24), in Figure $1 \mathrm{D}$, panel 3 we illustrate that while absorption and scattering limit the use of single-photon imaging with $488 \mathrm{~nm}$ excitation to image the entire 3-D PAS-stained normal heart (24), two-photon excitation of the same PAS at $780 \mathrm{~nm}$ excitation could produce the entire 3-D structure derived from the orthogonal cuts (Figure 1E).

We next tested the effectiveness of this technique using a widely studied $\mathrm{t} / \mathrm{t}$ heart having DCM (11). The results show that the $t / t$ heart had anomalous right and left ventricles (compare the volumes of the normal and t/t hearts in Figure 2, A, B, and G), dislocation of the most of the right ventricle (compare Figure 2, A and B, orthogonal section showing half heart, where the right ventricle from $t / t$ is barely seen), differences in the thickness of the right ventricle walls (Figure 2C), swelling (Figure 2D), and disarray of muscle sarcomeres (Figure 2, E and F). The quantitative evaluation of the volumes in 3-D between normal and $t / t$ analysis showed that the $t / t$ hearts scored higher in every aspect in terms of right and left ventricle volume and muscle and whole heart volume (Figure $2 \mathrm{G}$ ). It should be noted that the heart size is mainly determined by the muscle volume in both normal and t/t hearts, and it is amplified by the increased volumes of the ventricles in the $t / t$ heart (Figure 2G). This trend is the same in terms of 2-D morphometric measurements, except for the right ventricle wall diameter and length (Figure 3A), indicating that the overall size of the $t / t$ heart increase is contributed by enhanced ventricular volume more than the muscle area (Figure 3B). The left ventricle area increase is greater than the right ventricle, but the dislocation of the right ventricle is more substantial in the $t / t$ hearts when compared with normal hearts. The dislocation of the right ventricle in the $\mathrm{t} / \mathrm{t}$ hearts further demonstrates that an asymmetrical swelling of the hearts (which accounts for increased width and decreased length in t/t hearts) (Figure $3 \mathrm{~A}$ ) is accompanied by disruption in sarcomere structure in DCM. These results are in line with previously reported data using various histological, physiological, and pharmaco- logical methods to study the $t / t$ heart $(7,11,12,16)$.

We also tested this imaging technique for another purpose: to image the structure of decellularized murine hearts and to locate planted cells inside the organ, as this opens up a new way to study cellular behaviors such as proliferation and metastasis using decellularized hearts as tumor tissue substrates. Almost all cells grow within 3-D matrices, which vary in stiffness and components depending on the organ, while most in vitro cellular behavior studies are carried out on 2-D platforms because of the convenience of imaging and quantitative measurements. It is well-established that changes in the microenvironment can lead to completely different cellular behaviors $(26,27)$. In capturing in vivo cellular behaviors accurately in $3-D$, a decellularized scaffold derived from the same organ that the cells originate from is an ideal 3-D platform that closely mimics the real microenvironment in tissues. Figure 4 shows that when the decellularized hearts were placed in a cell culture, the cells from the culture could enter the scaffold and grow there within a natural extracellular matrix. Those cells could then be tracked with this technique after the experiment was terminated, when the heart was fixed and cleared with BABB. The single-photon spectral properties of PAS have been described before (22), but the two-photon excitation and usefulness of this dye have been shown here for the first time, to the best of our knowledge (Figure 4H). Our imaging technique, coupled with tissues grown in biologically derived matrices, may be useful in studies of cancer reversibility and carcinogenesis. It has been reported that misexpression of certain proteins and genes can transform normal epithelium into cancerous tissue (28), and at the same time, alteration in tissue matrices could induce cellular transformation and such transformed cells often exhibit genomic abnormalities (29). It would be interesting to investigate how cancer cells remodel their environment when planted in non-cancerous tissue matrices such as these decellularized heart scaffolds and how this remodeling affects gene expression in the cancer cells. 
The advantages of this method over existing techniques are the combination of homogenous labeling achieved by PAS staining of the entire heart and the ability to clear the heart with $\mathrm{BABB}$, rendering it optically clear/ transparent. Other advantages include whole-heart optical sectioning by the two-photon excitation, automated tiling and stitching of the images, and finally removing tile-scan artifacts by custom made MATLAB code. This makes wholemount imaging of the heart possible with minimum effort and allows fast, high-resolution 2-D and 3-D analysis.

\section{Author contributions}

M.S. conceived the idea. X.L., K.H.C., and B.L. isolated and provided the hearts. M.S. (NTG heart and scaffolds), B.S.S. (t/t heart), and V.A.S. (t/t heart) performed the clearing, microscopy, 3-D rendering and analysis, created volumes and analyzed 2-D and 3-D data. G.F. wrote the MATLAB code. M.T.A.S. and S.S. provided financial assistance and support. M.S. wrote the paper, which was edited by all of the authors.

\section{Acknowledgments}

We thank Claudia Lutz (Communication Department) and Carl R. Woese (IGB-UIUC) for assistance in editing the language and text. This work was supported by $\mathrm{NIH}$ grants R01 HL105826, K02 HL114749 (SS) and National Science Foundation ECCS grant 10-02165 (M.T.A.S.). Core facilities instrumentation and software at the Carl R. Woese Institute for Genomic Biology were used to collect data for this study. This paper is subject to the NIH Public Access Policy.

\section{Competing interests}

The authors declare no competing interests

\section{References}

1. Liu, X., K. Tobita, R.J. Francis, and C.W. Lo. 2013. Imaging techniques for visualizing and phenotyping congenital heart defects in murine models. Birth Defects Res. C Embryo Today 99:93-105.

2. Tobita, K., X. Liu, and C.W. Lo. 2010. Imaging modalities to assess structural birth defects in mutant mouse models. Birth Defects Res. C Embryo Today 90:176-184.
3. Schambach, S.J., S. Bag, L. Schilling, C. Groden, and M.A. Brockmann. 2010. Application of micro-CT in small animal imaging. Methods 50:2-13.

4. Dunmore-Buyze, P.J., E. Tate, F.L. Xiang, S.A. Detombe, Z. Nong, J.G. Pickering, and M. Drangova. 2014. Three-dimensional imaging of the mouse heart and vasculature using micro-CT and whole-body perfusion of iodine or phosphotungstic acid. Contrast Media Mol. Imaging 9:383-390.

5. Miller, C.E., R.P. Thompson, M.R. Bigelow, G. Gittinger, T.C. Trusk, and D. Sedmera. 2005. Confocal imaging of the embryonic heart: how deep? Microsc. Microanal. 11:216223.

6. Smith, R.M., A. Matiukas, C.W. Zemlin, and A.M. Pertsov. 2008. Nondestructive optical determination of fiber organization in intact myocardial wall. Microsc. Res. Tech. 71:510516.

7. Barefield, D., M. Kumar, J. Gorham, J.G. Seidman, C.E. Seidman, P.P. de Tombe, and S. Sadayappan. 2015. Haploinsufficiency of MYBPC3 exacerbates the development of hypertrophic cardiomyopathy in heterozygous mice. J. Mol. Cell. Cardiol. 79:234-243.

8. Weninger, W.J., S. Meng, J. Streicher, and G.B. Muller. 1998. A new episcopic method for rapid 3-D reconstruction: applications in anatomy and embryology. Anat. Embryol. (Berl.) 197:341-348.

9. Rosenthal, J., V. Mangal, D. Walker, M. Bennett, T.J. Mohun, and C.W. Lo. 2004. Rapid high resolution three dimensional reconstruction of embryos with episcopic fluorescence image

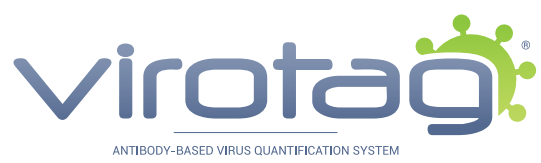

\section{Direct Antibody-Based Virus Quantification in Minutes!}

The ViroTag ${ }^{\circledR}$ detection system provides mission-critical data in real-time.

Eliminates the need for time-consuming plaque titer, qPCR and other methods when combined with the ViroCyt ${ }^{\circledR}$ Virus Counter ${ }^{\circledR} 3100$.

Immediate tracking of virus titer in your bioreactors to:

- Optimize growth conditions

- Maximize yields

- Identify problems sooner

Curious? Contact us at info@virocyt.com or visit www.virocyt.com to learn more!

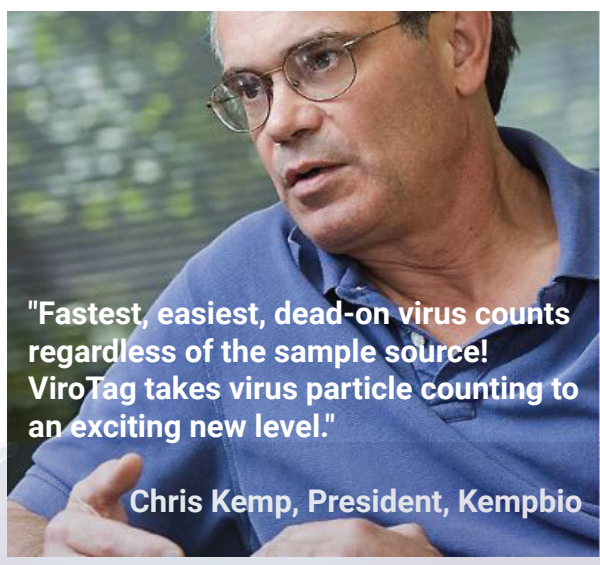


capture. Birth Defects Res. C Embryo Today 72:213-223.

10. Mohun, T.J. and W.J. Weninger. 2011. Imaging heart development using high-resolution episcopic microscopy. Curr. Opin. Genet. Dev. 21:573-578.

11. McConnell, B.K., K.A. Jones, D. Fatkin, L.H. Arroyo, R.T. Lee, O. Aristizabal, D.H. Turnbull, D. Georgakopoulos, et al. 1999. Dilated cardiomyopathy in homozygous myosin-binding protein-C mutant mice. J. Clin. Invest. 104:1771.

12. Sadayappan, S., J. Gulick, H. Osinska, L.A. Martin, H.S. Hahn, G.W. Dorn 2nd, R. Klevitsky, C.E. Seidman, et al. 2005. Cardiac myosin-binding protein-C phosphorylation and cardiac function. Circ. Res. 97:1156-1163.

13. Barefield, D. and S. Sadayappan. 2010. Phosphorylation and function of cardiac myosin binding protein- $\mathrm{C}$ in health and disease. J. Mol. Cell. Cardiol. 48:866-875.

14. Sadayappan, S., H. Osinska, R. Klevitsky, J.N. Lorenz, M. Sargent, J.D. Molkentin, C.E. Seidman, J.G. Seidman, and J. Robbins. 2006. Cardiac myosin binding protein C phosphorylation is cardioprotective. Proc. Natl. Acad. Sci. USA 103:16918-16923.

15. Sadayappan, S., J. Gulick, H. Osinska, D. Barefield, F. Cuello, M. Avkiran, V.M. Lasko, J.N. Lorenz, et al. 2011. A critical function for Ser-282 in cardiac Myosin binding protein-C phosphorylation and cardiac function. Circ. Res. 109:141-150.

16. Barefield, D., M. Kumar, P.P. de Tombe, and S. Sadayappan. 2014. Contractile dysfunction in a mouse model expressing a heterozygous MYBPC3 mutation associated with hypertrophic cardiomyopathy. Am. J. Physiol. Heart Circ. Physiol. 306:H807-H815.

17. Engler, A.J., S. Sen, H.L. Sweeney, and D.E. Discher. 2006. Matrix elasticity directs stem cell lineage specification. Cell 126:677-689.

18. Lin, P., W.C. Chan, S.F. Badylak, and S.N. Bhatia. 2004. Assessing porcine liver-derived biomatrix for hepatic tissue engineering. Tissue Eng. 10:1046-1053.

19. Klymkowsky, M.W. and J. Hanken. 1991. Whole-mount staining of Xenopus and other vertebrates. Methods Cell Biol. 36:419-441.

20. Gard, D.L. 1993. Confocal immunofluorescence microscopy of microtubules in amphibian oocytes and eggs. Methods Cell Biol. 38:241-264.

21. Zucker, R.M. 2006. Whole insect and mammalian embryo imaging with confocal microscopy: morphology and apoptosis. Cytometry A 69:1143-1152.

22. Changaris, D.G., J. Combs, and W.B. Severs. 1977. A microfluorescent PAS method for the quantitative demonstration of cytoplasmic 1,2-glycols. Histochemistry 52:1-15.

23. Fishman, A., S. Lew, M. Altaras, Y. Beyth, and J. Bernheim. 1998. A 30s PAS stain for frozen section analysis of surgical margins of vulvectomy in Paget's disease. Eur. J. Gynaecol. Oncol. 19:482-483.

24. Sivaguru, M., L. Mander, G. Fried, and S.W. Punyasena. 2012. Capturing the surface texture and shape of pollen: a comparison of microscopy techniques. PLoS ONE 7:e39129.
25. Sivaguru, M., G.A. Fried, C.A. Miller, and B.W. Fouke. 2014. Multimodal optical microscopy methods reveal polyp tissue morphology and structure in Caribbean reef building corals. J. Vis. Exp. 91:e51824.

26. Cukierman, E., R. Pankov, D.R. Stevens, and K.M. Yamada. 2001. Taking cell-matrix adhesions to the third dimension. Science 294:1708-1712.

27. Discher, D.E., P. Janmey, and Y.L. Wang. 2005. Tissue cells feel and respond to the stiffness of their substrate. Science 310:1139-1143.

28. Sternlicht, M.D., A. Lochter, C.J. Sympson, B. Huey, J.P. Rougier, J.W. Gray, D. Pinkel, M.J. Bissell, and Z. Werb. 1999. The stromal proteinase MMP3/stromelysin-1 promotes mammary carcinogenesis. Cell 98:137-146.

29. Lochter, A., Z. Werb, and M.J. Bissell. 1999. Transcriptional regulation of stromelysin-1 gene expression is altered during progression of mouse mammary epithelial cells from functionally normal to malignant. Matrix Biol. 18:455-467.

Received 05 May 2015; accepted 03 September 2015.

Address correspondence to Mayandi Sivaguru, Microscopy and Imaging Core Facility, Carl R. Woese Institute for Genomic Biology, University of Illinois at Urbana- Champaign, 1206 West Gregory Dr., Urbana, IL, 61801. E-mail: Sivaguru@illinois.edu

To purchase reprints of this article, contact: biotechniques@fosterprinting.com.

\section{Cyclic Dinucleotide STING Ligands}

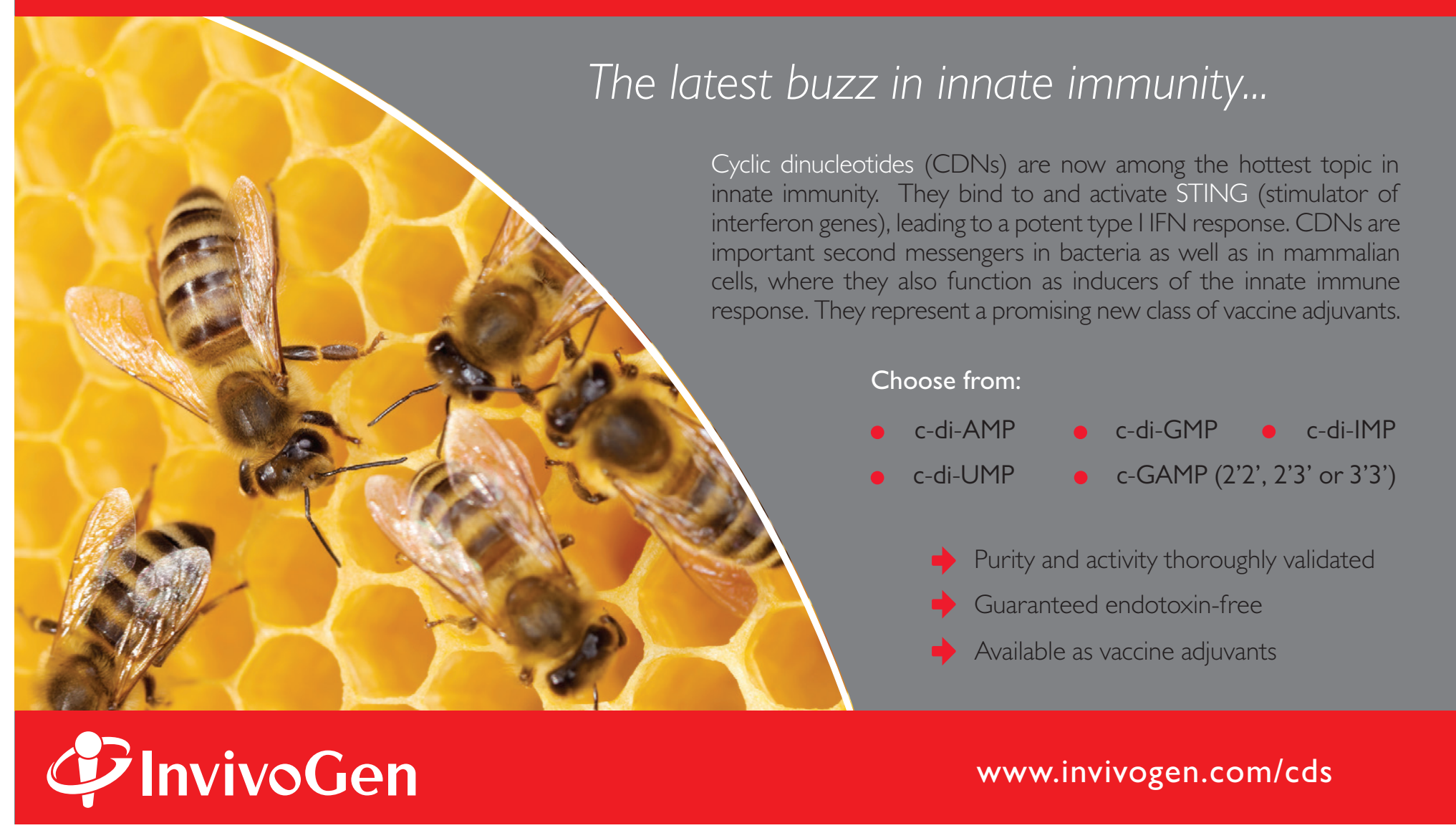

